H. Yokoi

Nagoya Math. J.

Vol. 102 (1986), 91-100

\title{
IMAGINARY BICYCLIC BIQUADRATIC FIELDS WITH THE REAL QUADRATIC SUBFIELD OF CLASS-NUMBER ONE
}

\author{
HIDEO YOKOI
}

It has been proved by A. Baker [1] and H. M. Stark [7] that there exist exactly 9 imaginary quadratic fields of class-number one. On the other hand, G.F. Gauss has conjectured that there exist infinitely many real quadratic fields of class-number one, and the conjecture is now still unsolved.

In connection with this Gauss' conjecture, we shall consider, in this paper, a real quadratic field $\boldsymbol{Q}(\sqrt{p})$ (prime $p \equiv 1 \bmod 4$ ) as a subfield of the imaginary bicyclic biquadratic field $K=\boldsymbol{Q}(\sqrt{p}, \sqrt{-q})$, which is a composite field of $\boldsymbol{Q}(\sqrt{p})$ with an imaginary quadratic field $\boldsymbol{Q}(\sqrt{-q})$ of class number one, and give various conditions for the class-number of $\boldsymbol{Q}(\sqrt{p})$ to be equal to one by using invariants of the relatively cyclic unramified extension $K / F$ over imaginary quadratic field $F=\boldsymbol{Q}(\sqrt{-p q})$.

After notation in Section 1, we shall summarize in Section 2 wellknown properties of a relatively cyclic extension and an unramified extension respectively, which we shall use in this paper. In Section 3 we shall consider the ideal class group of a cyclic unramified extension over a finite algebraic number field. Finally, we shall investigate in Section 4 the imaginary bicyclic biquadratic field $K=\boldsymbol{Q}(\sqrt{-q}, \sqrt{p})$, and give some conditions for the class-number of real quadratic subfield $\boldsymbol{Q}(\sqrt{p})$ to be equal to 1 .

\section{§1. Notation}

Generally, for an arbitrary finite abelian group $B$ and its subgroup $B^{\prime}$, the order of $B$ and the index of $B^{\prime}$ in $B$ are denoted by $|B|$ and $\left[B: B^{\prime}\right]$ respectively.

For an arbitrary number field $k$, the following notation is used

Received October 30, 1984. 
throughout this paper:

$E_{k}$ : the group of units of $k$

$C_{k}$ : the group of ideal classes of $k$

$h_{k}=\left|C_{k}\right|:$ the class-number of $k$

$\tilde{k}$ : the absolute or Hilbert class field of $k$.

For a finite Galois extension $K / F$ of a finite algebraic number field $F$ and the Galois group $G=\operatorname{Gal}(K / F)$, we shall denote by $H^{r}(G, B)$ the $r$-dimensional Galois cohomology group of $G$ acting on an abelian group $B$, and by $Q(B)$ the Herbrand quotient of $B$, i.e. $Q(B)=\left|H^{0}(G, B)\right| /\left|H^{1}(G, B)\right|$.

Furthermore, we shall use the following notation:

$\Pi e(\mathfrak{p})$ : the product of ramification exponents of all finite prime divisors $\mathfrak{p}$ of $F$ with respect to $K / F$

$\Pi e\left(\mathfrak{p}_{\infty}\right)$ : the product of ramification exponents of all infinite prime divisors $\mathfrak{p}_{\infty}$ of $F$ with respect to $K / F$

$\tilde{\Pi} e(\mathfrak{p})=\Pi e(\mathfrak{p}) \cdot \Pi e\left(\mathfrak{p}_{\infty}\right)$ : the product of ramification exponents of all finite and infinite prime divisors of $F$ with respect to $K / F$

$(\varepsilon)$ : the group of units of $F$

$(\eta)$ : the group of those units of $F$ which are norms of number of $K$

$A$ : the group of ambiguous classes of $C_{K}$ with respect to $K / F$

$a=|\boldsymbol{A}|:$ the ambiguous class number of $K / F$

$A_{0}$ : the group of classes of $C_{K}$ represented by ambiguous ideals with respect to $K / F$

$a_{0}=\left|A_{0}\right|$

$A_{F}$ : the group of classes of $C_{K}$ represented by ideals of $F$

$a_{F}=\left|A_{F}\right|$

$C_{F}^{0}$ : the group of those classes of $C_{F}$ whose ideals become principal in $K$

$h_{0}=\left|C_{F}^{0}\right|$

$N_{K / F}$ : the norm mapping with respect to $K / F$, and simultaneously the homomorphism from $C_{K}$ to $C_{F}$ induced by the norm mapping

$j=j_{K / F}$ : the homomorphism from $C_{F}$ to $C_{K}$ induced by extension of ideals

$N=j \circ N_{K / F}$ : the endomorphism of $C_{K}$ defined as composed mapping of $N_{K / F}$ and $j$.

\section{§2. Preliminary results}

In this section, we shall summarize several almost well-known 
results on a cyclic or an unramified extension, which we shall use in this paper.

Lemma $1{ }^{1{ }^{1}}$ Let $K / F$ be a finite Galois extension of a finite algebraic number field $F$, then

(1) $a_{0}=h_{F} \cdot \frac{\Pi e(\mathfrak{p})}{\left|H^{1}\left(G, E_{K}\right)\right|}$

(2) $H^{1}\left(G, E_{K}\right) \cong\left(A_{0}\right) /(\alpha)$ and $\left|H^{1}\left(G, E_{K}\right)\right| \equiv 0\left(\bmod h_{0}\right)$, where $\left(A_{0}\right)$ is the group of ambiguous principal ideals of $K$ with respect to $K / F$ and $(\alpha)$ is the group of principal ideals of $F$.

Lemma 2. ${ }^{2)}$ Let $K / F$ be a finite cyclic extension of a finite algebraic number field $F$, then

(3) $Q\left(C_{K}\right)=1, \quad Q\left(E_{K}\right)=\frac{\prod e\left(\mathfrak{p}_{\infty}\right)}{[K: F]}$

(4) $a=h_{F} \cdot \frac{\tilde{\Pi} e(\mathfrak{p})}{[K: F][\varepsilon: \eta]}=\left|N C_{K}\right| \cdot\left|H^{0}\left(G, C_{K}\right)\right|$

(5) $\frac{a}{a_{0}}=\left[\eta: N_{K / F}\left(E_{K}\right)\right], \quad \frac{a_{0}}{a_{F}}=\frac{h_{0} \cdot \Pi e(\mathfrak{p})}{\left|H^{1}\left(G, E_{K}\right)\right|}$

(6) $\tilde{\Pi} e(\mathfrak{p}) \equiv 0 \quad(\bmod [\varepsilon: \eta])$

Lemma $3{ }^{3)} \quad$ Let $K / F$ be a finite Galois unramified extension of a finite algebraic number field $F$, then

(7) $H^{1}\left(G, E_{K}\right) \cong C_{F}^{0}$

(8) $H^{2}\left(G, E_{K}\right) \cong A \mid A_{F}$

(9) $a=h_{F} \cdot \frac{\left|H^{2}\left(G, E_{K}\right)\right|}{\left|H^{1}\left(G, E_{K}\right)\right|}$.

\section{§3. Cyclic unramified extension}

Let $F$ be a finite algebraic number field, and $K$ be a finite cyclic unramified (in all finite and infinite prime divisors) extension field. For such extension $K / F$, we shall consider, in this section, the structure of the ideal class group $C_{K}$ of $K$ as Galois module.

Proposition 1. Let $K / F$ be a finite cyclic unramified extension of a finite algebraic number field $F$, then

1) For proofs, see Iwasawa [3], Yokoi [10].

2) For proofs, see Takagi [8, pp. 192-195], Yokoi [10].

3) For proofs, see Iwasawa [3]. 
(i) $a=\frac{h_{F}}{[K: F]}$, i.e. $\tilde{F}=K^{*}$,

where $K^{*}$ is the genus field with respect to $K / F$.

(ii) $h_{0}=\left|H^{1}\left(G, E_{K}\right)\right|=[K: F] \cdot\left[\eta: N_{K / F}\left(E_{K}\right)\right]$

(iii) $\left|H^{0}\left(G, C_{K}\right)\right|=\left|C_{F}^{0} \cap N_{K / F}\left(C_{K}\right)\right|$

(iv) $\left|H^{0}\left(G, C_{K}\right)\right| \equiv 0\left(\bmod \left|H^{\circ}\left(G, E_{K}\right)\right|\right)$, and $\left|H^{\circ}\left(G, C_{K}\right)\right|=\left|H^{\circ}\left(G, E_{K}\right)\right|$ if and only if $N C_{K}=A_{F}$

(v) any ambiguous class ideal of $K / F$ becomes principal in $\tilde{F}$.

Proof.

(i), (ii) See Yokoi [10]

(iii) See Kisilevsky [4]

(iv) By Lemma 2, (5), $\left[A: A_{0}\right]$ is equal to $\left[\eta: N_{K / F}\left(E_{K}\right)\right]$.

On the other hand, since $[\varepsilon: \eta]=1$ by Lemma 2 , (6), it holds $\left|H^{\circ}\left(G, E_{K}\right)\right|$ $=\left[\eta: N_{K / F}\left(E_{K}\right)\right]$, and so $\left[A: A_{0}\right]=\left|H^{0}\left(G, E_{K}\right)\right|$. Hence it is clear from $\left[A_{0}\right.$ : $\left.A_{F}\right]=1$ that

$$
\begin{aligned}
\left|H^{0}\left(G, C_{K}\right)\right| & =\left[A: A_{0}\right] \cdot\left[A_{0}: A_{F}\right] \cdot\left[A_{F}: N C_{K}\right] \\
& =\left|H^{0}\left(G, E_{K}\right)\right| \cdot\left[A_{F}: N C_{K}\right],
\end{aligned}
$$

which implies easily assertion (iv).

(v) See Terada [9], and cf (i).

Proposition 2. In the extension $K / F$, any two conditions of the following (i) $\sim$ (iii) are equivalent to each other:

(i) $h_{K}=a$, i.e. $C_{K}=A$

(ii) $\tilde{K}=K^{*}, \quad$ i.e. $C_{K}^{1-\sigma}=1$,

where $\sigma$ is a generator of the cyclic Galois group $G=\operatorname{Gal}(K / F)$.

(iii) $\operatorname{Ker}\left(N_{K / F}\right)=1$, i.e. $N_{K / F}: C_{K} \rightarrow C_{F}$ is monomorphic.

Proof. Since $\left[C_{F}: N_{K / F}\left(C_{K}\right)\right]=[K: F]$ and $a=h_{F} /[K: F]$ hold by class field theory and Proposition 1, (i) respectively, we get the following:

$$
\begin{aligned}
\operatorname{Ker}\left(N_{K / F}\right)=1 & \Longleftrightarrow\left|N_{K / F}\left(C_{K}\right)\right|=h_{K} \\
& \Longleftrightarrow h_{K}=h_{F} /[K ; F] \Longleftrightarrow h_{K}=a .
\end{aligned}
$$

On the other hand, it follows from $C_{K} / A \cong C_{K}^{1-\sigma}$ that

$$
h_{K}=a \Longleftrightarrow C_{K}=A \Longleftrightarrow C_{K}^{1-\sigma}=1 \Longleftrightarrow \tilde{K}=K^{*} .
$$

Proposition 3. In the extension $K / F$, any two conditions of the following (i) $\sim$ (iv) are equivalent to each other: 
(i) $a=a_{0}$, i.e. $A=A_{0}$

(ii) $\left[\eta: N_{K / F}\left(E_{K}\right)\right]=1$

(iii) $H^{0}\left(G, E_{K}\right)=1$

(iv) $\left|H^{1}\left(G, E_{K}\right)\right|=h_{0}=[K: F]$

Proof. (i) $\Longleftrightarrow$ (ii) It is evident by Lemma 2, (5) that (i) is equivalent to (ii).

(ii) $\Longleftrightarrow$ (iii) Since $K / F$ is a cyclic unramified extension, we get $[\varepsilon: \eta]$ $=1$ immediately by Lemma 2, (6), and so

$$
\left|H^{0}\left(G, E_{K}\right)\right|=[\varepsilon: \eta] \cdot\left[\eta: N_{K / F}\left(E_{K}\right)\right]=\left[\eta: N_{K / F}\left(E_{K}\right)\right] .
$$

Hence

$$
\left|H^{0}\left(G, E_{K}\right)\right|=1 \text { if and only if }\left[\eta: N_{K / F}\left(E_{K}\right)\right]=1 .
$$
(iv).

(ii) $\Longleftrightarrow$ (iv) It is clear by Proposition 1 , (ii) that (ii) is equivalent to

Proposition 4. In the extension $K / F$, any two conditions of the following (i) $\sim$ (iii) are equivalent to each other:

(i ) $C_{F}=C_{F}^{0} \times N_{K / F}\left(C_{K}\right)$

(ii) $\operatorname{Ker}(N)=\operatorname{Ker}\left(N_{K / F}\right)$

(iii) $H^{0}\left(G, C_{K}\right)=1$

Proof. (i) $\Longrightarrow$ (ii) Since $N=j \circ N_{K / F}$, it holds $\operatorname{Ker}\left(N_{K / F}\right) \subset \operatorname{Ker}(N)$ in general. If $C_{F}=C_{F}^{0} \times N_{K / F}\left(C_{K}\right)$, then $C_{F} \cap N_{K / F}\left(C_{K}\right)=1$ holds, and hence for any $C$ in $\operatorname{Ker}(N)$ we get $N_{K / F}(C) \in C_{F}^{0} \cap N_{K / F}\left(C_{K}\right)$, and so $C \in \operatorname{Ker}\left(N_{K / F}\right)$. Therefore we get $\operatorname{Ker}(N) \subset \operatorname{Ker}\left(N_{K / F}\right)$.

(ii) $\Longrightarrow$ (iii) If $\operatorname{Ker}\left(N_{K / F}\right)=\operatorname{Ker}(N)$, then for any $C^{\prime}$ in $C_{F}^{0} \cap N_{K / F}\left(C_{K}\right)$, it holds

$$
\phi \neq N_{K / F}^{-1}\left(C^{\prime}\right) \in N_{K / F}^{-1}\left(C_{F}^{0}\right)=\operatorname{Ker}(N)=\operatorname{Ker}\left(N_{K / F}\right), \text { and so } C^{\prime}=1 .
$$

Hence we get $C_{F}^{0} \cap N_{K / F}\left(C_{K}\right)=1$, from which follows $H^{\circ}\left(G, C_{K}\right)=1$ by Proposition 1, (iii).

(iii) $\Longrightarrow$ (i) If $H^{0}\left(G, C_{K}\right)=1$, then $C_{F}^{0} \cap N_{K / F}\left(C_{K}\right)=1$ holds by Proposition 1, (iii). On the other hand, by class field theory $\left|N_{K / F}\left(C_{K}\right)\right|=h_{F} /[K: F]$ holds, and also by Proposition 1, (ii),

$$
\left|C_{F}^{0}\right|=h_{0} \equiv 0 \quad(\bmod [K: F])
$$

holds. Hence we get $C_{F}=C_{F}^{0} \times N_{K / F}\left(C_{K}\right)$. 
Corollary. In the extension $K / F$, if any one of 3 conditions in Proposition 4 is satisfied, then each of 4 conditions in Proposition 3 is also satisfied.

Proof. This assertion is an immediate consequence of Proposition 1, (iv), Proposition 3 and Proposition 4.

\section{§4. Imaginary bicyclic biquadratic field}

Let $p$ be a prime congruent to $1 \bmod 4$, and $q$ be 1,2 or a prime congruent to $-1 \bmod 4$. Put $k_{1}=\boldsymbol{Q}(\sqrt{-q}), k_{2}=\boldsymbol{Q}(\sqrt{p}), F=\boldsymbol{Q}(\sqrt{-p q})$ and $K=\boldsymbol{Q}(\sqrt{-q}, \sqrt{p})$. Then, applying the results of Section 3, we shall consider, in this section, the structure of the ideal class group $C_{K}$ of $K$ as Galois module with respect to $K / F$, and under the assumption that the class-number $h_{1}$ of $k_{1}$ is equal to 1 , we shall give some kinds of conditions for the class-number $h_{2}$ of $k_{2}$ to be equal to 1 .

Theorem 1. Let $p$ be a prime congruent to $1 \bmod 4$, and $q$ be 1,2 or a prime congruent to $-1 \bmod 4 . \quad$ Put $F=\boldsymbol{Q}(\sqrt{-p q})$ and $K=\boldsymbol{Q}(\sqrt{-q}, \sqrt{p})$. Then, $K / F$ is a cyclic unramified extension of degree 2, and moreover the following (i) $\sim(\mathrm{v})$ hold:

(i ) $K^{*}=\tilde{F}$

(ii) $h_{K}=h_{F} \cdot \frac{h_{1} \cdot h_{2}}{2}$

(iii) $H^{0}\left(G, E_{K}\right)=1$

(iv) $a=a_{0}$, i.e. $A=A_{0}$

(v) $h_{0}=2$

Here, $h_{1}$ and $h_{2}$ are the class-number of quadratic number fields $k_{1}=\boldsymbol{Q}(\sqrt{-q})$ and $k_{2}=\boldsymbol{Q}(\sqrt{p})$ respectively.

Proof. In the imaginary bicyclic biquadratic field $K=Q(\sqrt{-q}, \sqrt{p})$, the ramified finite primes are only $p$ and $q$ (or $\left.2^{4}\right)$, and their ramification exponents with respect to $K / \boldsymbol{Q}$ are equal to theirs with respect to $K / F$ respectively (all of them are equal to 2). Hence $K / F$ is unramified.

(i) $\tilde{F}=K^{*}$ follows immediately from Proposition 1.

(ii) Since $p \equiv 1(\bmod 4)$, the fundamental unit $\varepsilon_{p}$ of $k_{2}$ has norm -1 . Hence, we know first

$$
h_{K}=\frac{h_{1} \cdot h_{2} \cdot h_{F}}{2} \quad \text { (see, for example, Brown and Parry [2]). }
$$

4) In the special case of $q=1$, there is choosen 2 instead of $q$. 
(iii) Since $N_{K / F}\left(\varepsilon_{p}\right)=N_{k_{2}}\left(\varepsilon_{p}\right)=-1$, we get

$$
(\varepsilon)= \pm 1=N_{K / F}\left(E_{K}\right) \text {. }
$$

Hence

$$
H^{0}\left(G, E_{K}\right) \cong(\varepsilon) / N_{K / F}\left(E_{K}\right)=1 \text {. }
$$

(iv), (v) Both $a=a_{0}$ and $h_{0}=2$ are immediate consequences of Proposition 3 and the above assertion (iii).

Corollary. Let $K / F$ be as in Theorem 1 , then

(i ) $a=a_{0}=h_{F} / 2$

(ii) $H^{1}\left(G, E_{K}\right)$ is a cyclic group of order 2 .

Proof. These two assertions are immediate consequences of Theorem 1 and Proposition 1.

Theorem 2. If the class-number $h_{1}$ of $\boldsymbol{Q}(\sqrt{-q})$ is equal to 1 , then any two conditions of the following $(\mathrm{i}) \sim(\mathrm{v})$ are equivalent to each other:

(i) the class-number $h_{2}$ of $\boldsymbol{Q}(\sqrt{p})$ is equal to 1

(ii) $h_{K}=a$, i.e. $C_{K}=A$

(iii) $\tilde{K}=K^{*}$, i.e. $C_{K}^{1-\sigma}=1$

(iv) $N_{K / F}: C_{K} \rightarrow C_{F}$ is monomorphic, i.e. $\operatorname{Ker}\left(N_{K / F}\right)=1$

(v) $j: C_{F} \rightarrow C_{K}$ is epimorphic, i.e. $j\left(C_{F}\right)=C_{K}$.

Proof. (i) $\Longleftrightarrow$ (ii) By Theorem 1, it follows from the assumption that

$$
h_{2}=1 \quad \text { if and only if } h_{K}=h_{F} / 2 \text {. }
$$

On the other hand, since $a=h_{F} / 2$ by Proposition 1, (i), we have that

$$
h_{2}=1 \quad \text { if and only if } h_{K}=a .
$$

(ii) $\Longleftrightarrow$ (iii) Since $C_{K} / A \cong C_{K}^{1-\sigma}$ and $\left[C_{K} ; C_{K}^{1-\sigma}\right]=\left[K^{*}: K\right]$, it is clear that

$$
C_{K}=A \Longleftrightarrow C_{K}^{1-\sigma}=1 \Longleftrightarrow \tilde{K}=K^{*} .
$$

(ii) $\Longleftrightarrow$ (iv) Since $C_{K}$ is finite,

$$
\operatorname{Ker}\left(N_{K / F}\right)=1 \quad \text { if and only if }\left|N_{K / F}\left(C_{K}\right)\right|=h_{K} .
$$

On the other hand, since $\left[C_{F}: N_{K / F}\left(C_{K}\right)\right]=2$ by class field theory,

$$
\left|N_{K / F}\left(C_{K}\right)\right|=h_{K} \quad \text { if and only if } h_{K}=h_{F} / 2 \text {, }
$$

which is equivalent to $h_{K}=a$. 
(ii) $\Longleftrightarrow$ (v) Since $C_{F} / C_{F}^{0} \cong j\left(C_{F}\right)$ and $\left|C_{F}^{0}\right|=2$ by Theorem 1, we get

$$
\left|j\left(C_{F}\right)\right|=\left[C_{F}: C_{F}^{0}\right]=h_{F} / 2 .
$$

Hence, for $\boldsymbol{C}_{K} \supset j\left(\boldsymbol{C}_{F}\right)$ we have

$$
C_{K}=j\left(C_{F}\right) \Leftrightarrow h_{K}=h_{F} / 2 \Longleftrightarrow h_{K}=a .
$$

Consequently, $j$ is epimorphic if and only if $h_{K}=a$.

Proposition 5. If the class-number $h_{1}$ of $\boldsymbol{Q}(\sqrt{-q})$ is equal to 1 , then it is necessary for the class-number $h_{2}$ of $\boldsymbol{Q}(\sqrt{p})$ to be equal to 1 that the following conditions (i) $\sim$ (iii) are satisfied:

(i) $H^{0}\left(G, C_{K}\right)=1$ or cyclic group of order 2

(ii) 2 rank $s$ of the ideal class group $C_{K}$ of $K$ is equal to 0 or 1

(iii) all ideals of $K$ become principal in $\tilde{F}$.

Proof. (i ) By Theorem 1, (v), it follows from $C_{F}^{0} \supset C_{F}^{0} \cap N_{K / F}\left(C_{K}\right)$ that

$$
\left|C_{F}^{0} \cap N_{K / F}\left(C_{K}\right)\right|=1 \text { or } 2 \text {, }
$$

and hence we know by Proposition 1, (iii)

$$
\left|H^{\circ}\left(G, C_{K}\right)\right|=1 \text { or } 2 \text {. }
$$

(ii) By Theorem 2 it holds $C_{K}=A$, which implies

$$
N C_{K}=N A=A^{2}=C_{K}^{2} .
$$

Thus we get

$$
\left|H^{\circ}\left(G, C_{K}\right)\right|=\left[A: N C_{K}\right]=\left[C_{K}: C_{K}^{2}\right]=2^{s},
$$

and hence the assertion (ii) implies $s=0$ or 1 .

(iii) The assertion (iv) follows immediately from $C_{K}=A$ by Proposition 1 , (v).

Proposition 6. Under the assumption $h_{1}=1$, if we assume moreover $h_{2}=1$, then any two conditions of the following (i) $\sim$ (iv) are equivalent to each other:

$$
\text { (i) }\left(\frac{q}{p}\right)=-1 \text {, }
$$

where (-) is the Legendre-Jacobi-Kronecker symbol.

(ii) $h_{F} \not \equiv 0(\bmod 4)$, i.e. $2 \| h_{F}$

(iii) 2 rank $s$ of $C_{K}$ is equal to 0 , i.e. $\left(h_{K}, 2\right)=1$ 
(iv) $H^{n}\left(G, C_{K}\right)=1$ for any integer $n$.

Proof. (i ) $\Longleftrightarrow$ (ii) It is an immediate consequence of Rédei and Reichardt's theorem that

$$
h_{F} \not \equiv 0(\bmod 4) \text { if and only if }\left(\frac{p}{q}\right)=-1
$$

(see Rédei and Reichardt [6]) .

(ii) $\Longleftrightarrow$ (iii) Since assumption $h_{1}=h_{2}=1$ implies $h_{K}=h_{F} / 2$ by Theorem 1, (ii), it is clear that

$$
\left(h_{K}, 2\right)=1 \text { if and only if } h_{F^{\prime}} \neq \equiv 0(\bmod 4) .
$$

(iii) $\Longleftrightarrow$ (iv) By Theorem 2, assumption $h_{1}=h_{2}=1$ implies $C_{K}=A$. On the other hand,

$$
\left(h_{K}, 2\right)=1 \text { if and only if } C_{K}^{2}=C_{K} .
$$

Hence, if $\left(h_{K}, 2\right)=1$, then we get

$$
N C_{K}=N A=A^{2}=C_{K}^{2}=C_{K}=A,
$$

which shows $H^{\circ}\left(G, C_{K}\right) \cong A / N C_{K}=1$, and by Lemma 2 , (3) $H^{n}\left(G, C_{K}\right)=1$ holds for any integer $n$. Conversely, if $H^{n}\left(G, C_{K}\right)=1$ holds for any integer $n$, then in particular $H^{0}\left(G, C_{K}\right)=1$ implies $A=N C_{K}$. Hence we get

$$
C_{K}^{2}=A^{2}=N A=N C_{K}=A=C_{K},
$$

which shows $\left(h_{K}, 2\right)=1$.

Proposition 7. Under the assumption $h_{1}=1$, if the endomorphism $N$ of $C_{K}$ is epimorphic or monomorphic, the following conditions (i) $\sim$ (iii) are satisfied:

(i) $h_{2}=1$

(ii) $H^{n}\left(G, C_{K}\right)=1$ for any integer $n$

(iii) 2 rank $s$ of $C_{K}$ is equal to 0 ,

$$
\text { i.e. }\left(h_{K}, 2\right)=1
$$

Proof. Since $C_{K}$ is a finite abelian group, the following conditions $\left(1^{\circ}\right) \sim\left(3^{\circ}\right)$ for the endomorphism $N$ of $C_{K}$ are equivalent to each other:

$\left.1^{\circ}\right) \quad N$ is epimorphic

$\left.2^{\circ}\right) \quad N$ is monomorphic

$\left.3^{\circ}\right) \quad N$ is automorphic. 
In this case, it follows from $C_{K}=N C_{K}$ that $C_{K}=A=N C_{K}$ holds, which implies $2^{s}=\left[C_{K}: C_{K}^{2}\right]=1$ because $C_{K}^{2}=A^{2}=N A=N C_{K}=C_{K}$. Thus we know $s=0$, which is assertion (iii).

Moreover, by Theorem 2, $C_{K}=A$ implies $h_{2}=1$, which is assertion (i).

On the other hand, $A=N C_{K}$ implies $H^{0}\left(G, C_{K}\right) \cong A / N C_{K}=1$, and hence by Lemma 2 , (3) we get $H^{n}\left(G, C_{K}\right)=1$ for any integer $n$. Thus, we can complete the proof of Proposition 7 .

Finally, we give some examples.

\begin{tabular}{|r|r|r|r|r|r|r|}
\hline \multicolumn{1}{|c|}{$p$} & $q$ & $h_{1}$ & $h_{2}$ & $h_{F}$ & $a$ & $h_{K}$ \\
\hline 5 & 1 & 1 & 1 & 2 & 1 & 1 \\
17 & 2 & 1 & 1 & 4 & 2 & 2 \\
13 & 2 & 1 & 1 & 6 & 3 & 3 \\
41 & 1 & 1 & 1 & 8 & 4 & 4 \\
53 & 3 & 1 & 1 & 10 & 5 & 5 \\
229 & 3 & 1 & 3 & 26 & 13 & 39 \\
\hline
\end{tabular}

\section{REFERENCES}

[1] A. Baker, Linear forms in the logarithms of algebraic numbers, Mathematika, 13 (1966), 204-216.

[2] E. Brown and C. J. Parry, The imaginary bicyclic biquadratic fields with classnumber 1, J. Reine Angew. Math., 260 (1973), 118-120.

[ 3 ] K. Iwasawa, A note on the group of units of an algebraic number field, J. Math. Pures Appl., 35 (1956), 189-192.

[4] H. Kisilevsky, Some results related to Hilbert's Theorem 94, J. Number Theory, 2 (1970), 199-206.

[ 5 ] S. Kuroda, Über den Dirichletschen Körper, J. Fac. Sci. Imp. Univ. Tokyo, Sec. I, 4 (1943), 383-406.

[6] L. Rédei and H. Reichardt, Die Anzahl der durch 4 teilbaren Invarianten der Klassengruppe beliebigen quadratischen Zahlkörpers, J. Reine Angew. Math., 170 (1933), 69-74.

[7] H. M. Stark, A complete determination of the complex quadratic fields of classnumber one, Michigan Math. J., 14 (1967), 1-27.

[ 8 ] T. Takagi, Algebraic number Theory (Japanese), Iwanami, Tokyo (1948).

[ 9 ] F. Terada, A principal ideal theorem in the genus fields, Tôhoku Math. J., 23-4 (1971), 697-718.

[10] H. Yokoi, On the class number of a relatively cyclic number field, Nagoya Math. J., 29 (1967), 31-44.

Department of Mathematics

College of General Education

Nagoya University

Chikusa-ku, Nagoya 464

Japan 